\title{
Impact of the neoclassical distribution function on turbulent impurity and momentum fluxes: fluid model and gyrokinetic simulations
}

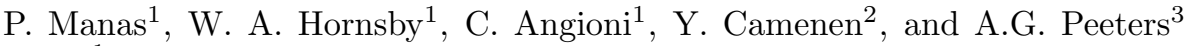 \\ 1 Max-Planck-Institut für Plasmaphysik, D-85748 Garching, Germany \\ ${ }^{2}$ Aix-Marseille Université, CNRS, PIIM UMR7345, 13397 Marseille, France \\ 3 Department of Physics, University of Bayreuth, 95440 Bayreuth, Germany
}

\begin{abstract}
The impact of the neoclassical background on turbulent impurity transport is investigated by means of gyrokinetic simulations supported by fluid equations. The latter are derived, using a Laguerre polynomials expansion of the first order neoclassical distribution function, and analytical expressions of the turbulent momentum flux and impurity transport coefficients are assessed. Comparisons of gyrokinetic simulations including this neoclassical background (coupling between the codes GKW and NEO) and the fluid model are used to identify the main mechanisms behind the modification of the turbulent transport channels and benchmark the numerical implementation. These mechanisms include a modification of the parallel dynamics of the main ions and direct contributions stemming from the asymmetry in the parallel velocity space of the neoclassical distribution function. The latter which is found dominant for turbulent impurity transport, increases with increasing collisionality, $R / L_{T_{i}}, R / L_{n}$, impurity mass, safety factor and aspect ratio. These contributions to momentum and impurity fluxes are also found to depend on the directions of the toroidal magnetic field and plasma current.
\end{abstract}

Keywords:

\section{INTRODUCTION}

Understanding impurity radial transport in magnetic confinement devices is of importance to avoid detrimental effects like impurity accumulations. In these devices, neoclassical transport stemming from collisions in toroidal geometry and turbulent transport stemming from fluctuations in the electromagnetic potential at Larmor radius scales coexist. The predicted impurity density profiles resulting from an independent numerical treatment of neoclassical and turbulent transport have been successfully compared to experimental light and heavy impurity profiles in several cases [1-4]. Other recent studies reported poor agreement between theory and experiments for impurity transport whether it is for tokamaks $[5,6]$ or stellarators $[7,8]$ where the respective weights of neoclassical and turbulent transport are intrinsically different.

It has been realised recently that the cross-talk between the neoclassical and turbulent channels could be of importance [9-11]. The impact of the neoclassical equilibrium on turbulent momentum transport has been explored in details in [12-16] and found to be significant compared to other sources of momentum flux [16] such as the Coriolis drift and the flux surface up-down asymmetry. Turbulent momentum and impurity transport being not completely disconnected, in particular with respect to the role of symmetry breaking mechanisms [17, 18], these results motivated a study of the impact of the neoclassical equilibrium distribution function on turbulent impurity transport. To this end, two approaches are adopted: the derivation of a fluid model including this correction and numerical simulations using the coupled codes NEO $[19,20]$ and GKW[22].

The former approach allows us to underline physical ingredients and qualitative behaviours with varying typ- ical plasma parameters, the latter provides quantitative results on this new turbulent impurity transport mechanism. The objectives are twofold: highlight the parameter space for which the impact of the neoclassical equilibrium on impurity transport is relevant and benchmark the implementation of the neoclassical equilibrium in the GKW code made in [16]. While the emphasis is on turbulent impurity transport, similarities in deriving analytical expressions for the main ion and trace impurities transport enable a simultaneous treatment of the turbulent impurity and parallel momentum transport. We underline that the present study focuses on the impact of neoclassical flows on turbulence and does not consider the impact of turbulence on neoclassical transport.

The paper is organised as follows. Section II comprises the definition of the neoclassical background in use for the derivation of the fluid model as well as the modified gyrokinetic equation. In section III the perturbed density, parallel velocity and temperature fluid equations are expressed including the neoclassical background. From these equations the turbulent momentum flux and the impurity transport coefficients are derived. Gyrokinetic simulations are performed in section IV and qualitative comparisons with the analytical expressions are performed to stress both the model and the implementation of the neoclassical background in GKW. Then simulations encompassing a large plasma parameter space provide information on where the impact of the neoclassical background correction on turbulent fluxes is expected to be large. Finally, conclusions are given in section $\mathrm{V}$. 


\section{NEOCLASSICAL BACKGROUND CORRECTION TO THE GYROKINETIC EQUATION}

In this section, the gyrokinetic equation including the neoclassical background distribution function is introduced. An analytical solution of the drift-kinetic equation is used to describe the neoclassical deviation from the Maxwellian background and decomposed into Laguerre polynomials. The fluid model derived in section IV only retains the first term of the decomposition (the parallel neoclassical flow) but the implementation in the gyrokinetic code GKW [16] can retain any number of Laguerre polynomials, as set in the neoclassical code NEO.

\section{A. Analytical first order neoclassical distribution function}

The solution to the first order (in $\rho_{*}=\rho_{i} / R$ with $\rho_{i}$ the ion Larmor radius and $R$ the major radius of the torus) drift kinetic equation can be decomposed as a sum of Laguerre polynomials [23]:

$$
F_{n e o, a}=F_{M, a} \frac{m_{a} v_{\|}}{T_{a}} \sum_{j=0}^{\infty} u_{a, j} L_{j}^{3 / 2}\left(x^{2}\right)
$$

with the total distribution function $F_{\text {tot }}$ described by:

$$
F_{\text {tot }}=F_{M}+F_{\text {neo }}
$$

with $F_{M}=n /\left(\pi^{3 / 2} v_{t h}^{3}\right) \exp \left\{-\left[\left(v_{\|}-v_{\phi}\right)^{2}-v_{\perp}^{2}\right] / v_{t h}^{2}\right\}$ a Maxwellian (with $v_{\phi}=B_{t} / B R\left(\omega_{\phi}(r)-\Omega\right)$, $\Omega$ the frame angular frequency and $\omega_{\phi}$ the plasma angular frequency at a given radial position $r$, related to the lowest order radial electric field $\left.\mathrm{d} \phi_{-1} / \mathrm{d} r[20,21]\right)$. The subscript $a$ denotes a given species, $n$ the density, $m$ the mass, $v_{\|}$ the velocity parallel to the magnetic field, $T$ the temperature, $B_{t}$ the toroidal magnetic field strength, $B$ the magnetic field strength, $R$ the major radius, $L_{j}^{3 / 2}$ the modified Laguerre polynomials and $x=v / v_{t h, a}$, with $v_{t h, a}=\sqrt{2 T_{a} / m_{a}}$ the thermal velocity. The coefficients $u_{a, j}$ are defined by:

$$
u_{a, j}=\frac{c_{j}}{n_{a}} \int v_{\|} L_{j}^{3 / 2} F_{n e o, a} \mathrm{~d}^{3} \mathbf{v}
$$

with

$$
c_{j}=\frac{3 \times 2^{j} j !}{(2 j+3) ! !}
$$

Throughout the paper, 'neoclassical background' will refer to $F_{n e o}$.

Usually two polynomials are kept in this expansion, consisting of the parallel flow $\left(U_{\|}\right)$and parallel heat flow $\left(q_{\|}\right)$[23-25], though particular physical problems require higher number of polynomials [25]:

$$
F_{n e o, a}=F_{M, a} \frac{m_{a} v_{\|}}{T_{a}}\left[U_{\|}-\frac{2 q_{\|}}{5 p_{a}}\left(\frac{5}{2}-x^{2}\right)\right]
$$

with $p$ the pressure. In this equation $U_{\|}$is not the total flow and the lowest order toroidal flow is contained in the Maxwellian as described above.

In the following, only the first term is retained, that is the neoclassical parallel flow $U_{\|}$, to derive the fluid model and underline the physical mechanisms behind turbulent transport modifications via the neoclassical background. The neglect of the parallel heat flow and higher order terms in the fluid model is addressed in Appendix A by comparing gyrokinetic simulations retaining from 1 to 5 Laguerre polynomials for the neoclassical background distribution function.

In this paper, particular interest is given to impurity (more specifically on roto-diffusion [17]) and momentum transport as they both rely on parallel symmetry breaking $[12,17,18]$. To this end the neoclassical first order distribution function is needed for impurities as well as for the main ions. Following Eq. (25) and (27) of [24] to express the neoclassical parallel flow in terms of the main ions background gradients for trace impurity species, the neoclassical background correction can be recast as:

$$
\begin{aligned}
F_{n e o, a} & =s_{B} s_{J} F_{M, a} \rho_{*} \frac{m_{a}}{m_{i}} \frac{B_{t}}{B_{p}} \frac{v_{\|}}{v_{t h, i}}\left[\frac{R}{L_{T_{i}}}\left(1-K_{a}\right)\right. \\
& \left.+\frac{R}{L_{n}}-R \frac{e}{T_{i}} \frac{\partial \phi_{0}}{\partial r}\right]
\end{aligned}
$$

with $a$ denoting either the impurity $(I)$ or the main ions $(i), R / L_{T}$ and $R / L_{n}$ the normalised logarithmic temperature and density gradients respectively, $B_{p}$ the poloidal magnetic field strength, $r$ the minor radius, $\phi_{0}$ the zeroth order electrostatic potential, $s_{B}$ and $s_{J}$ the signs of the toroidal magnetic field and plasma current respectively (positive values correspond to the counterclockwise direction when viewed from above), $e$ the elementary charge and $K_{a}$ the dimensionless flow coefficient depending on collisionality and on the species $a$ $[20,24,27]$. In the following we consider $\partial \phi_{0} / \partial r$ to be negligible. This is justified for cases with small centrifugal effects and temperature anisotropies, which is the case throughout this study.

The neoclassical deviation from the Maxwellian is first order in $\rho_{*}$ but is enhanced by the impurity mass, the ratio $B_{t} / B_{p} \sim q / \epsilon$ and the Maxwellian background gradients. Equation (6) already encapsulates all the important dependencies numerically investigated in section IV. 


\section{B. Gyrokinetic equation and neoclassical background correction}

The gyrokinetic equation for the distribution function $F$ of species $a[28]$ is given by:

$$
\frac{\partial F_{a}}{\partial t}+\frac{\mathrm{d} \mathbf{X}}{\mathrm{d} t} \cdot \nabla F_{a}+\frac{\mathrm{d} v_{\|}}{\mathrm{d} t} \frac{\partial F_{a}}{\partial v_{\|}}=0
$$

with the following equations of motion:

$$
\begin{aligned}
\frac{\mathrm{d} \mathbf{X}}{\mathrm{d} t} & =v_{\|} \mathbf{b}+\mathbf{v}_{\mathbf{D}}+\mathbf{v}_{\mathbf{E}} \\
\frac{\mathrm{d} v_{\|}}{\mathrm{d} t} & =\frac{1}{m_{a} v_{\|}} \frac{\mathrm{d} \mathbf{X}}{\mathrm{d} t} \cdot(Z e \mathbf{E}-\mu \nabla B)
\end{aligned}
$$

with $\mathbf{v}_{\mathbf{D}}$ denoting the $\nabla B$, curvature and Coriolis drifts and $\mathbf{v}_{\mathbf{E}}$ the $E \times B$ drift. Centrifugal and electromagnetic effects are neglected in the derivation.

In the framework of the $\delta f$ approximation $\left(F=F_{b}+\delta f\right.$ with $F_{b}$ the background distribution function, $\delta f$ the perturbed distribution function with the ordering: $\delta f / F_{b} \ll$ $1)$, valid at low $\rho_{*}$, this equation can be recast as:

$$
\frac{\partial \delta f}{\partial t}+\frac{\mathrm{d} \mathbf{X}}{\mathrm{d} t} \cdot \nabla \delta f+\frac{\mathrm{d} v_{\|}}{\mathrm{d} t} \frac{\partial \delta f}{\partial v_{\|}}=S
$$

with $S$ the source term provided by the background distribution function $F_{b}$. To study deviations from the Maxwellian background due to the neoclassical flows, one can express $F_{b}$ as in Eq. (2), that is, also considering the neoclassical background $F_{\text {neo }}$ described by Eq. $(6)$. The left hand side of the gyrokinetic equation being not modified by the neoclassical background distribution function (it is only modified via the neoclassical electrostatic potential entering in the nonlinear and the trapping terms), we focus on the right hand side source term $S$. Though retained in numerical simulations, the neoclassical electrostatic potential is dropped in the rest of the derivation (negligible contributions compared to the perturbed turbulent electrostatic potential).

Rewriting $S$, using the definitions for the neoclassical background yields:

$$
\begin{aligned}
S & =\frac{\mathrm{d} \mathbf{X}}{\mathrm{d} t} \cdot\left\{( F _ { n e o , a } + F _ { M , a } ) \frac { 1 } { R } \left[\frac{R}{L_{n_{a}}} \nabla r+\frac{R}{L_{T_{a}}}\right.\right. \\
& \left.\left(\frac{E}{T_{a}}-\frac{3}{2}\right) \nabla r-2 R \nabla \omega_{\phi} \frac{R B_{t}}{B v_{t h, a}^{2}} v_{\|}-\frac{R Z_{a} e \nabla\langle\delta \phi\rangle}{T_{a}}\right] \\
& \left.-F_{M, a} \nabla H-\frac{F_{n e o}}{m_{a} v_{\|}^{2}}(Z e \nabla\langle\delta \phi\rangle-\mu \nabla B)\right\}
\end{aligned}
$$

with $E$ the species kinetic energy $1 / 2 m_{a}\left(v_{\|}^{2}+v_{\perp}^{2}\right), \delta \phi$ the perturbed electrostatic potential, the brackets \langle\rangle denoting the gyro-average and $H$ defined to be the terms in front of the Maxwellian in equation 6:

$$
H=s_{B} s_{J} \rho_{*} \frac{m_{a}}{m_{i}} \frac{B_{t}}{B_{p}} \frac{v_{\|}}{v_{t h, i}}\left[\frac{R}{L_{T_{i}}}\left(1-K_{a}\right)+\frac{R}{L_{n}}\right]
$$

Second derivatives as well as dominant terms such as $\left(R / L_{T_{i}}\right)^{2}$ enter through the $\nabla H$ term.

The slab-like geometry employed in $[17,28]$ is used and the following definitions are recalled for completeness:

$$
\mathbf{B}=s_{B} B \mathbf{e}_{\mathbf{y}}, \quad \nabla B=-\frac{B}{R} \mathbf{e}_{\mathbf{x}}, \quad \boldsymbol{\Omega}=\Omega \mathbf{e}_{\mathbf{z}}
$$

with $\left(\mathbf{e}_{\mathbf{x}}, \mathbf{e}_{\mathbf{y}}, \mathbf{e}_{\mathbf{z}}\right)$ forming a right-handed orthogonal coordinate system. Using these definitions $\mathrm{d} \mathbf{X} / \mathrm{d} t$ can be written as:

$$
\begin{aligned}
\frac{\mathrm{d} \mathbf{X}}{\mathrm{d} t} & =s_{B} v_{\|} \mathbf{e}_{\mathbf{y}}+\frac{s_{B}}{Z e B R}\left(m_{a} v_{\|}^{2}+\mu B\right) \mathbf{e}_{\mathbf{x}} \times \mathbf{e}_{\mathbf{y}} \\
& +s_{B} \frac{\mathbf{e}_{\mathbf{y}} \times \nabla\langle\delta \phi\rangle}{B}+s_{B} \frac{2 m_{a} v_{\|}}{Z e B} \Omega \mathbf{e}_{\mathbf{z}}
\end{aligned}
$$

In this simple slab-like geometry, the drifts depend only on the direction of the toroidal magnetic field $\left(s_{B}\right)$ whereas in more complex geometries they can also depend on $s_{J}$. Finally, the perturbed quantities $\delta f$ and $\delta \phi$ are written as a sum over Fourier modes:

$$
\delta A=\sum_{k_{\|}, k_{z}} \delta \hat{A}_{k_{\|}, k_{z}} \exp \left(\mathrm{i} k_{\|} y+\mathrm{i} k_{z} z-\mathrm{i} \omega t\right)
$$

with $A$ being either $f$ or $\phi, k_{\|}=k_{y}$ and $k_{z}$ the parallel and perpendicular to the magnetic field wave vectors respectively. From now on, the subscripts of the Fourier amplitudes $\delta \hat{A}$ are dropped and only one mode is considered. Using Eq. (11), (12) and (13), neglecting Finite Larmor Radius (FLR) effects and using $\omega_{D}=-k_{z} T_{a} /(e B R)$, one arrives at the following expression for the source term $S$ :

$$
\begin{aligned}
S & =s_{B}\left(F_{n e o}+F_{M}\right) \delta \hat{\phi} \frac{e}{T_{a}}\left\{-Z k_{\|} v_{\|}+\frac{\omega_{D}}{T_{a}}\left(m_{a} v_{\|}^{2}+\mu B\right)\right. \\
& +2 \omega_{D} \frac{m_{a}}{T_{a}} R \Omega v_{\|}-\omega_{D}\left[\frac{R}{L_{n_{a}}}+\frac{R}{L_{T_{a}}}\left(\frac{E}{T_{a}}-\frac{3}{2}\right)-\right. \\
& \left.\left.2 R^{2} \nabla \omega_{\phi} \frac{v_{\|}}{v_{t h, a}^{2}}\right]\right\}+s_{B} \omega_{D} \frac{e}{T_{a}} \delta \hat{\phi} F_{M, a} \frac{\partial H}{\partial r}-s_{B} F_{n e o} \frac{e}{T_{a}} \frac{\delta \hat{\phi}}{m_{a} v_{\|}^{2}} \\
& {\left[Z_{a} T_{a} v_{\|} k_{\|}-\omega_{D}\left(m_{a} v_{\|}^{2}+2 \mu B\right)-2 \omega_{D} R \Omega m_{a} v_{\|}\right] }
\end{aligned}
$$

Using equation 6 and neglecting gradients of the equilibrium magnetic field and of the dimensionless flow coefficient $K_{a}$, one obtains for $\partial H / \partial r$ :

$$
\begin{gathered}
R \frac{\partial H}{\partial r}=s_{B} s_{J} \frac{m_{a}}{m_{i}} \rho_{*} \frac{B_{t}}{B_{p}} \frac{v_{\|}}{v_{t h, i}}\left[( 1 - K _ { a } ) \left(\frac{R^{2}}{T_{i}} \frac{\partial^{2} T_{i}}{\partial r^{2}}-\right.\right. \\
\left.\left.\left(\frac{R}{L_{T_{i}}}\right)^{2}\right)+\frac{R^{2}}{n} \frac{\partial^{2} n}{\partial r^{2}}-\left(\frac{R}{L_{n}}\right)^{2}\right]
\end{gathered}
$$


In the present study, $B_{t} / B_{p}$ and the collisionality are assumed to vary slowly with the minor radius and their radial gradients are neglected in Eq. (15) (but are retained in the numerical simulations). These terms could have a significant impact close the plasma edge and can be added straightforwardly to the fluid model if need be. It is recalled here that the fluid model does not retain the poloidal variations of the neoclassical flows (low field side model), which is another difference with the numerical simulations.

\section{IMPACT OF THE NEOCLASSICAL BACKGROUND ON IMPURITY AND} MOMENTUM TRANSPORT: FLUID MODEL

In this section, a fluid model is derived by taking moments of Eq. (14) as in $[17,28]$ and the turbulent im- purity and momentum fluxes are computed. Analytical dependencies of the neoclassical background induced turbulent transport are then highlighted.

\section{A. Fluid equations}

Using a Maxwellian closure, one can write the first three moment equations in the form:

$$
\begin{aligned}
\tilde{n_{a}} & \left(s_{B} \omega_{N}+\frac{2}{Z}\right)-\tilde{v_{a}}\left(k_{\|, N}-4 x_{a} \frac{u}{Z}\right)+\frac{2}{Z} \tilde{T}_{a}=-\hat{\phi} \frac{T_{e}}{T_{a}}\left\{s_{B} s_{J} \rho_{*} \frac{B_{t}}{B_{p}} \frac{m_{a}}{m_{i}}\left[\frac{R}{L_{T_{i}}}\left(1-K_{a}\right)+\frac{R}{L_{n}}\right]\right. \\
& \left.\left(-u^{\prime}+3 u-\frac{Z}{x_{a}} k_{\|, N}\right)-\frac{R}{L_{n_{a}}}+2\right\} \\
\tilde{v_{a}} & \left(s_{B} \omega_{N}+\frac{4}{Z}\right)-\left(\tilde{n_{a}}+\tilde{T_{a}}\right)\left(\frac{k_{\|, N}}{2}-\frac{2}{Z} x_{a} u\right)=-\hat{\phi} \frac{T_{e}}{T_{a}}\left\{-\frac{Z}{2} k_{\|, N}+2 x_{a} u-x_{a} u^{\prime}+s_{B} s_{J} \frac{x_{a}}{2} \rho_{*} \frac{B_{t}}{B_{p}} \frac{m_{a}}{m_{i}}\left[\left(1-K_{a}\right)\right.\right. \\
& \left.\left.\left(\frac{R^{2}}{T_{i}} \frac{\partial^{2} T_{i}}{\partial r^{2}}-\frac{R}{L_{T_{i}}}+\frac{R}{L_{T_{i}}}\left(7-\frac{R}{L_{n_{a}}}+2 \frac{R}{L_{T_{a}}}\right)\right)+\left(\frac{R^{2}}{n} \frac{\partial^{2} n}{\partial r^{2}}-\frac{R^{2}}{L_{n}}+\frac{R}{L_{n}}\left(7-\frac{R}{L_{n_{a}}}+2 \frac{R}{L_{T_{a}}}\right)\right)\right]\right\} \\
\tilde{T}_{a} & \left(3 s_{B} \omega_{N}+\frac{14}{Z}\right)+\frac{4}{Z} \tilde{n_{a}}-2 \tilde{v_{a}}\left(k_{\|, N}-\frac{4}{Z} x_{a} u\right)=-\hat{\phi} \frac{T_{e}}{T_{a}}\left\{4-3 \frac{R}{L_{T_{a}}}-s_{B} s_{J} \rho_{*} \frac{B_{t}}{B_{p}} \frac{R}{m_{i}}\left(1-K_{a}\right)+\frac{R}{L_{T_{i}}}\right] \\
& {\left.\left[\frac{Z}{x_{a}} k_{\|, N}-7 u+2 u^{\prime}\right]\right\} }
\end{aligned}
$$

with $x_{a}=v_{t h, i} / v_{t h, a}$ and the following normalisations and notations:

$$
\begin{aligned}
k_{\|, N} & =k_{\|} \frac{v_{t h, a}}{\omega_{D}}, \quad \hat{\phi}=\frac{e}{T_{e}} \delta \hat{\phi}, \quad \omega_{N}=\frac{\omega_{R}+\mathrm{i} \gamma}{\omega_{D}} \\
\gamma_{N} & =\frac{\gamma}{\omega_{D}}, \quad \omega_{D}=-\frac{k_{z} T_{a}}{e B R}, \quad u=\frac{R \Omega}{v_{t h, i}} \\
u^{\prime} & =\frac{R^{2}}{v_{t h, i}} \frac{\partial \omega_{\phi}}{\partial r}
\end{aligned}
$$

$\omega_{R}$ and $\gamma$ are the real frequency and growth rate respectively of the mode. Setting neoclassical corrections to zero, i.e. $\rho_{*} \rightarrow 0$, one recovers equations of [17]. In Eq. (16), (17) and (18), new terms involving the velocity space integrals of $v_{\|} F_{n e o}$ contribute as new sources for the moments of the distribution function $\delta f$. For even moments such as density and temperature, these terms stem from drifts which are odd in $v_{\|}$such as the Coriolis drift, the parallel streaming and the $E \times B$ advection in the background toroidal rotation gradient. On the other hand, for odd moments such as the perturbed parallel velocity, even parity of the drifts are now required for a nonzero contribution of the neoclassical background. These drifts are now the $E \times B$ advection in the background density and temperature gradients, the curvature and $\nabla B$ drifts. In other words adding the first order neoclassical background to the standard Maxwellian involves new terms related to symmetry breaking mechanisms $\left(u^{\prime}\right.$, $\left.u, k_{\|}\right)$directly in the perturbed density and temperature equations. This leads for instance to the generation of a nonzero roto-diffusion directly from the perturbed density equation without any coupling with the perturbed parallel velocity equation.

It is also shown that the gradient of the neoclassical flows, i.e. second derivatives and $\left(R / L_{T_{i}}\right)^{2}$ terms enter only in the pertubed parallel velocity equation. It is then 
expected to have more impact on the turbulent momentum flux than on the turbulent impurity transport (in the case of low coupling, i.e. $k_{\|, N}-4 x_{a} \frac{u}{Z} \sim 0$ ).

To highlight the physical ingredients responsible for modifications of the turbulent momentum and impurity fluxes via the neoclassical background, we consider the cases $k_{\|, N}-4 x_{a} \frac{u}{Z}=0$ and $\tilde{T}=0$. The former condition is relevant for sufficiently small values of $u$ and $u^{\prime}$ (for which the related $k_{\|}$will be small). On the contrary the condition $\tilde{T}=0$ is artificial and justified a posteriori via gyrokinetic simulations showing no effect of the neoclassical equilibrium on thermo-diffusion. Furthermore it does not prevent the study of the main mechanisms at play. The $\tilde{T}=0$ condition can be relaxed to obtain interesting dependencies on the mode frequency (section IV). Cases with $k_{\|, N}-4 x_{a} \frac{u}{Z} \neq 0$ will also be investigated via numerical simulations in section IV. With these two assumptions, the fluid model equations are:

$$
\begin{aligned}
\tilde{n_{a}} & \left(s_{B} \omega_{N}+\frac{2}{Z}\right)=-\hat{\phi} \frac{T_{e}}{T_{a}}\left\{2-\frac{R}{L_{n_{a}}}-s_{B} s_{J} \rho_{*} \frac{B_{t}}{B_{p}} \frac{m_{a}}{m_{i}}\right. \\
& {\left.\left[\frac{R}{L_{T_{i}}}\left(1-K_{a}\right)+\frac{R}{L_{n}}\right] u^{\prime}\right\} } \\
\tilde{v_{a}} & \left(s_{B} \omega_{N}+\frac{4}{Z}\right)=-s_{B} s_{J} \hat{\phi} \frac{T_{e}}{T_{i}} \frac{\rho_{*} x_{a}}{2} \frac{m_{a}}{m_{i}} \frac{B_{t}}{B_{p}}\left\{\left(1-K_{a}\right)\right. \\
& {\left[\frac{R^{2}}{T_{i}} \frac{\partial^{2} T_{i}}{\partial r^{2}}-\left(\frac{R}{L_{T_{i}}}\right)^{2}+\frac{R}{L_{T_{i}}}\left(7-\frac{R}{L_{n_{a}}}+2 \frac{R}{L_{T_{a}}}\right)\right]+} \\
& \left.\frac{R^{2}}{n} \frac{\partial^{2} n}{\partial r^{2}}-\left(\frac{R}{L_{n}}\right)^{2}+\frac{R}{L_{n}}\left(7-\frac{R}{L_{n_{a}}}+2 \frac{R}{L_{T_{a}}}\right)\right\}
\end{aligned}
$$

These two equations are interesting as they describe in the most reduced way the impact of the neoclassical background on the turbulent fluxes aside from a modification of the symmetry of the eigenfunction $\left(k_{\|}=0\right)$. This is particularly suitable for impurity transport (as traces) which is the main topic of this study. For momentum transport, the neoclassical background necessarily induce a nonzero $k_{\|}$which then limits the extent of Eq. (23). This feature is discussed in section IV.

\section{B. Turbulent fluxes}

Using the same definitions for the turbulent particle $(\Gamma)$ and momentum (П) fluxes as in [17]:

$$
\begin{array}{r}
\Gamma=\frac{1}{4} n_{0} v_{t h, i} \frac{T_{e}}{T_{i}} \sum_{k_{z}, k_{\|}} k_{z} \rho_{i} \exp (2 \gamma t) \Im\left(\hat{\phi}^{\dagger} \tilde{n}\right) \\
\Pi=\frac{s_{B}}{4} n_{0} v_{t h, i} \frac{T_{e}}{T_{i}} \sum_{k_{z}, k_{\|}} k_{z} \rho_{i} \exp (2 \gamma t) \Im\left(\hat{\phi}^{\dagger} \tilde{v}_{\|}\right)
\end{array}
$$

and using Eq. (22) and (23) for given $k_{z}$ and $k_{\|}$, one obtains:

$$
\begin{aligned}
\Gamma_{Z} & =\frac{s_{B}}{4} n_{Z} v_{t h, i}\left(\frac{T_{e}}{T_{i}}\right)^{2} k_{z} \rho_{i} \exp (2 \gamma t) \frac{|\hat{\phi}|^{2}}{\left|\omega_{N}+\frac{2}{Z}\right|^{2}} \gamma_{N}\left\{2-\frac{R}{L_{n_{Z}}}-s_{B} s_{J} \rho_{*} \frac{B_{t}}{B_{p}} \frac{m_{Z}}{m_{i}}\left[\frac{R}{L_{T_{Z}}}\left(1-K_{a}\right)+\frac{R}{L_{n_{i}}}\right] u^{\prime}\right\} \\
\Pi_{i} & =\frac{s_{J} s_{B}}{4} n v_{t h, i}\left(\frac{T_{e}}{T_{i}}\right)^{2} k_{z} \rho_{i} \exp (2 \gamma t) \frac{|\hat{\phi}|^{2}}{\left|\omega_{N}+4\right|^{2}} \gamma_{N}\left(\frac { \rho _ { * } } { 2 } \frac { B _ { t } } { B _ { p } } \left\{\left(1-K_{a}\right)\left[\frac{R^{2}}{T_{i}} \frac{\partial^{2} T_{i}}{\partial r^{2}}-\left(\frac{R}{L_{T_{i}}}\right)^{2}+\frac{R}{L_{T_{i}}}\left(7-\frac{R}{L_{n_{i}}}+2 \frac{R}{L_{T_{i}}}\right)\right]\right.\right. \\
& \left.\left.+\left[\frac{R^{2}}{n_{i}} \frac{\partial^{2} n_{i}}{\partial r^{2}}-\left(\frac{R}{L_{n_{i}}}\right)^{2}+\frac{R}{L_{n_{i}}}\left(7-\frac{R}{L_{n_{i}}}+2 \frac{R}{L_{T_{i}}}\right)\right]\right\}\right)
\end{aligned}
$$

For further comparisons with the momentum flux computed in GKW, a $s_{B}$ has been added to Eq 25 to match the corresponding definition in the code.

The terms proportional to $\rho_{*}$ in Eq. (26) and (27) are the contributions to the impurity and momentum fluxes driven by the neoclassical background. The momentum flux driven by the neoclassical background depends on the second order derivatives of the temperature and density. As for the impurity particle flux, it can be further decomposed into a convective and a diffusive part (see e.g [33]):

$$
\Gamma_{z}=n_{Z} \frac{D}{R}\left(\frac{R}{L_{n_{z}}}+C_{T} \frac{R}{L_{T_{z}}}+C_{u} u^{\prime}+C_{p}\right)
$$

with $D$ the diffusivity, $C_{T}$ the thermo-diffusion coefficient, $C_{u}$ the roto-diffusion coefficient and $C_{p}$ the curvature pinch. In the case of trace impurities this relation is strictly linear with respect to the gradients $R / L_{n_{z}}$, $R / L_{T_{z}}$ and $u^{\prime}$. From Eq. (26) and (28) one can then 
express the transport coefficients $C_{u}$ and $C_{p}$ :

$$
\begin{aligned}
C_{u}^{N C} & =s_{B} s_{J} \rho_{*} \frac{B_{t}}{B_{p}} \frac{m_{Z}}{m_{i}}\left[\frac{R}{L_{T_{i}}}\left(1-K_{I}\right)+\frac{R}{L_{n_{i}}}\right] \\
C_{p} & =-2
\end{aligned}
$$

The superscript ${ }^{N C}$ denotes the contribution of the neoclassical background to the roto-diffusion in contrast to the standard contributions stemming from toroidal rotation $u$ and its gradient $u^{\prime}$. Thermo-diffusion is not discussed analytically as coupling with the perturbed temperature equation has been neglected [34].

As already discussed, $C_{u}^{N C}$ does not require a couping between the perturbed density and parallel velocity, unlike the roto-diffusion driven by a Maxwellian background. Furthermore this expression highlights important dependencies on plasma parameters. Indeed $C_{u}^{N C}$ increases with collisionality (due to the neoclassical parallel flow coefficient $\left.K_{a}\right), R / L_{T_{i}}, R / L_{n}, m_{z}$ and $B_{t} / B_{p} \sim$ $q / \epsilon$. Finally, the direction of the toroidal magnetic field and plasma current, made explicit in Eq. (6) via $s_{B}$ and $s_{J}$, is expected to change the direction of $C_{u}^{N C}$ and the neoclassical background induced momentum flux.

In summary, using the following hypotheses: one Laguerre polynomial to describe the neoclassical background, slab-like geometry, fluid moments, no FLR effects, $k_{\|, N}-4 x_{a} u / Z \sim 0, \tilde{T}=0, \nabla K_{a}=0$, no poloidal variations of the neoclassical flows, one arrives at the following results:

- The neoclassical background induced roto-diffusion coefficient $C_{u}^{N C}$ increases with the following parameters: $R / L_{n}, R / L_{T_{i}}, 1-K_{a}$ and the species mass.

- The turbulent momentum flux features quadratic relations with $R / L_{T_{i}}$ and $R / L_{n}$.

- Radial second derivatives of the density and temperature backgrounds are important ingredients in the momentum flux generation via the neoclassical background radial gradient.

- Both $\Pi_{i}$ and $C_{u}^{N C}$ are increasing with increasing safety factor $q$ and decreasing $\epsilon$ (due to the term $\left.B_{t} / B_{p}\right)$ and are expected to be dependent on the plasma current and/or toroidal magnetic field directions.

In the next section these points are investigated by means of gyrokinetic simulations.

\section{GYROKINETIC SIMULATIONS AND NEOCLASSICAL BACKGROUND CORRECTION}

To compare predictions of Eq. (27) and (29) with numerical simulations, the flux tube, local version of GKW is used. The neoclassical background $F_{n e o}$ is computed using NEO and is then added to the Maxwellian background used in GKW (the neoclassical electrostatic potential is also taken into account). A description of the NEO-GKW coupling can be found in [16].

First, the quantitative impact of the neoclassical background on the turbulent momentum flux and rotodiffusion is numerically assessed for two trace impurities (Carbon and Tungsten) at increasing collisionalities. The distinction between $k_{\|}=0$ and $k_{\|} \neq 0$ cases is underlined. Then a complete investigation of the parametric dependencies (including the collisionality, $R / L_{n}, R / L_{T}$, $q / \epsilon$, the impurity mass and the signs of the toroidal magnetic field and plasma current) is performed and the numerical results are compared to Eq. (27) and (29). Finally, the impact of second derivatives and mode frequencies on the neoclassical background induced momentum flux and roto-diffusion are explored.

The following electrostatic simulations are performed using the Cyclone base case [30] at the normalised poloidal wave number $k_{\theta} \rho_{i}=0.42$ (peak of the ITG linear growth rate spectrum). Parameters for this case are recalled in Table I. Circular flux surfaces are specified using the Miller geometry [31] in both NEO and GKW. The normalised ion Larmor radius $\rho_{i} / R$ employed throughout the paper is set to $2.5 \times 10^{-3}$ which is a typical value found in medium sized tokamaks. In this work, the direct impact of collisions on the turbulent fields is modelled in GKW with the pitch-angle scattering operator. The main ions are deuterium nuclei and electrons are treated kinetically.

\begin{tabular}{|c|c|c|c|c|c|c|}
\hline$R / L_{T_{i}}$ & $R / L_{T_{e}}$ & $T_{e} / T_{i}$ & $R / L_{n}$ & $q$ & $\hat{s}$ & $\epsilon$ \\
\hline 6.9 & 6.9 & 1 & 2.2 & 1.4 & 0.8 & 0.18 \\
\hline
\end{tabular}

TABLE I: Cyclone parameters used in linear simulations. $\hat{s}$ corresponds to the magnetic shear $(\hat{s}=r / q \partial q / \partial r)$.

To compute the first order neoclassical distribution function a total of 5 Laguerre and 17 Legendre polynomials are used together with the linearised Fokker-Planck collision operator in NEO. The same number of polynomials is kept in GKW. NEO simulations at five radial locations are used to compute the radial derivative of the neoclassical distribution function, with the values of $R / L_{n}$ and $R / L_{T_{i}}$ at the central point corresponding to the values in Table I. To start with, the second order derivatives are chosen to be zero.

Convergence tests of the linear simulations have been performed, yielding the following gridsizes: $n_{\mu}=16$, $n_{v_{\|}}=64, n_{s}=30, n_{x}=21$ for the number of points in the magnetic moment, parallel velocity, parallel direction, and radial wavenumbers respectively.

The following definition of the normalised quasilinear ion parallel momentum flux is employed for qualitative comparisons with Eq 27:

$$
\Pi_{N}=\Pi_{i} \frac{R}{n_{e} m_{i} v_{t h, i}^{2} \rho_{*}^{2}} \frac{1}{|\phi|^{2}}
$$




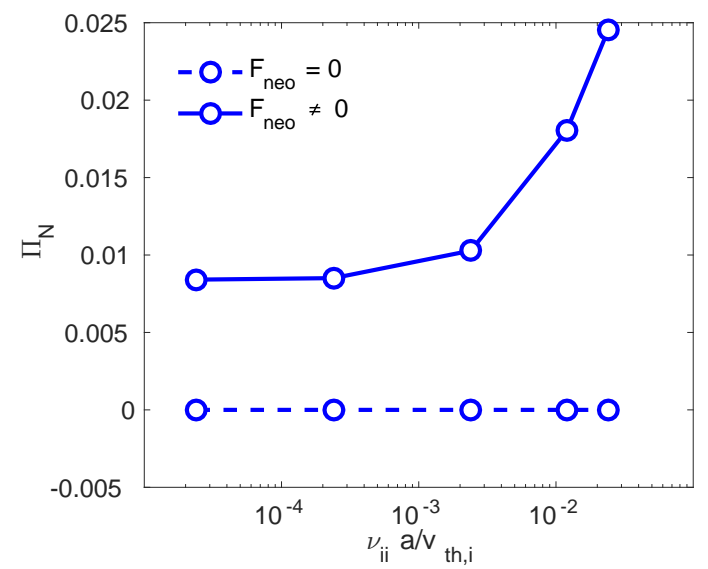

FIG. 1: Normalised ion momentum flux versus the normalised ion-ion collisionality with and without the neoclassical background correction.

where $\Pi_{i}$ is the contravariant flux surface averaged radial flux:

$$
\Pi_{i}=\left\langle\int \mathrm{d}^{3} \mathbf{v} s_{B} m v_{\|} \mathbf{v}_{\mathbf{E}} \cdot \nabla r \delta f\right\rangle_{s}
$$

A first set of linear simulations, varying the ion-ion collisionality is performed in Fig. 1 and 2 to assess the impact of the neoclassical background on the turbulent ion momentum flux and the impurity transport coefficients. The normalised toroidal rotation $u=R \Omega / v_{t h, i}$ has been set to zero together with its gradient $u^{\prime}=-R^{2} / v_{t h, i}\left(\partial \omega_{\phi} / \partial r\right)$ (for the main ions and kinetic electrons), insuring no symmetry breaking mechanisms aside from the one introduced via the neoclassical background. A set of orthogonal background gradients is applied to several trace impurities (similarly to [32]) including a nonzero $u^{\prime}$ to compute transport coefficients. The effects on two distinct trace species, namely for carbon and tungsten, are investigated. In both figures, 1 and 2 , the neoclassical correction $F_{\text {neo }}$ is applied to all of the species, that is the main ions (Deuterium), electrons and the trace impurity species.

In Fig. 1 the ion momentum flux is shown to be strictly zero with $F_{\text {neo }}=0$, i.e. no neoclassical background correction, as expected from the choice of parameters (no toroidal rotation and corresponding gradient for the main species). An increase with the ion-ion collisionality is observed when $F_{\text {neo }} \neq 0$ stemming from the collisionality dependence of the neoclassical poloidal flow. At low collisionalities a nonzero residual turbulent ion momentum flux remains, consistent with $[13,14]$ where contributions of $F_{\text {neo }}$ to the turbulent ion momentum flux in different collisionality regimes are also discussed. No reversal of the momentum flux is observed for this case (no background $E \times B$ rotation, the only source of momentum flux is the neoclassical background) in agreement with the results presented in [13].
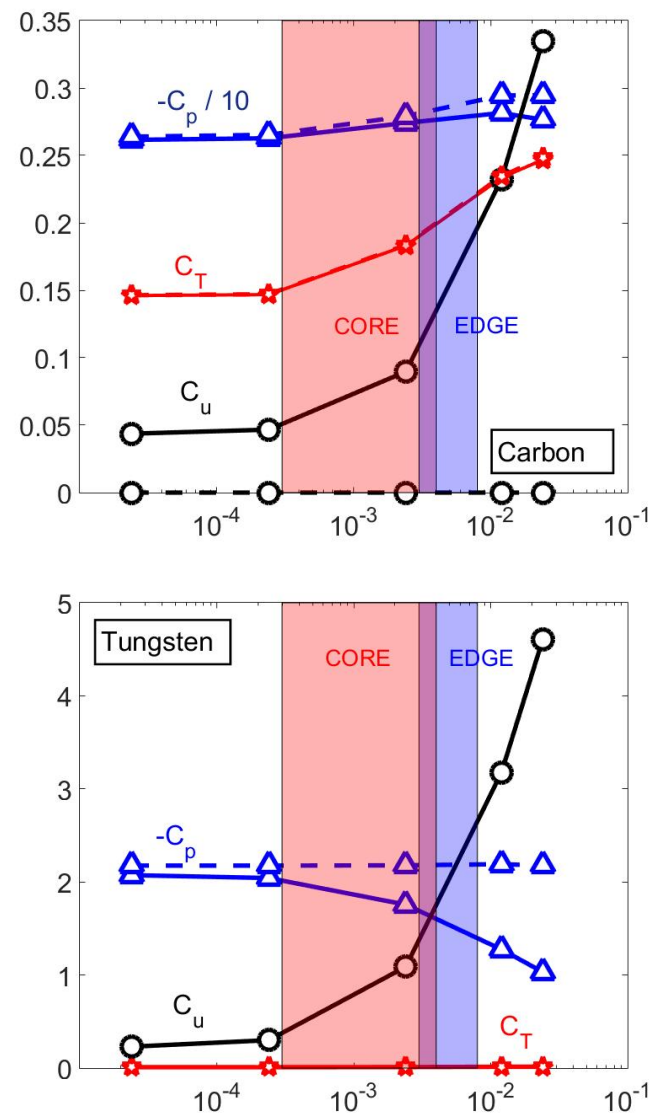

FIG. 2: (color online) Transport coefficients $C_{p}$ (triangles), $C_{T}$ (pentagrams) and $C_{u}$ (circles) versus the normalised ion-ion collisionality for carbon (a) and tungsten (b) trace impurities. The curvature pinch $C_{p}$ has been rescaled in the first panel. Simulations with/without the neoclassical background correction are represented with solid/dashed lines. Typical core and edge collisionality windows found in present tokamaks are also highlighted.

In Fig. 2, the roto-diffusion in the case $F_{\text {neo }}=0$ is again strictly zero (absence of symmetry breaking inherent to this convective mechanism [17]). Including the neoclassical background correction yields a nonzero $C_{u}$, increasing with the collisionality, similarly to the ion momentum flux. The curvature pinch $C_{p}$ also presents modifications due to $F_{\text {neo }}$ but not as marked as for the rotodiffusion coefficient. For carbon, $C_{u}$ can become comparable to the thermo-diffusion coefficient at high collisionality, but remains smaller than $C_{p}$. For tungsten, however, $C_{u}$ can be comparable in magnitude to the curvature pinch due to the mass dependence in the neoclassical background distribution function. This strong effect on tungsten convection could be experimentally relevant at the edge of NBI heated plasmas where the collisionality is high and the toroidal rotation gradient u' typically around unity, making the roto-diffusion contribution dominant. It is also recalled here that the amplitude of the roto-diffusion coefficient generated by the neoclassical equilibrium does not depend solely on col- 


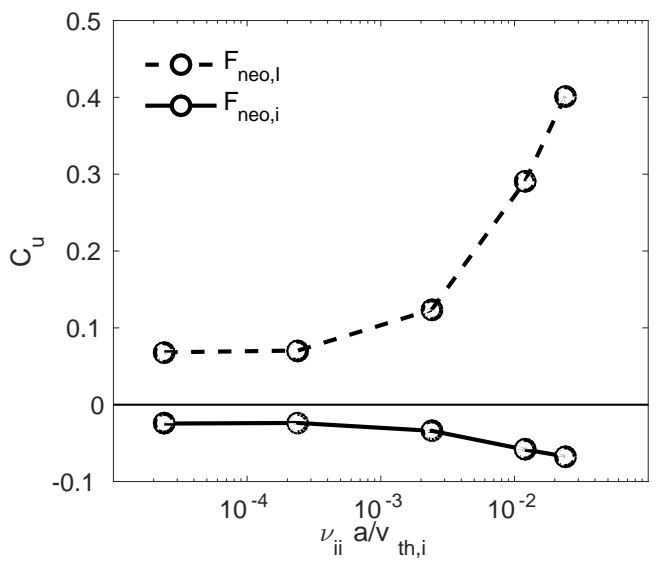

FIG. 3: Roto-diffusion coefficient $C_{u}$ for trace carbon versus the normalised ion-ion collisionality. Neoclassical background corrections are applied to either the main ions $\left(F_{n e o, i}\right)$ or to the trace impurities $\left(F_{n e o, I}\right)$.

lisionality and other regimes where this mechanism is quantitatively important may exist. Finally, no modifications of the thermo-diffusion coefficient are observed in the range of collisionalities used. The correction on $C_{p}$ being small and the one on $C_{T}$ being negligible compared to the changes introduced on $C_{u}$, special emphasis is given to the latter. It is important to note however that the small corrections on the curvature pinch yield a reduced inward convection, leading to a concomitant effect (increasing outward roto-diffusion and reduced inward $C_{p}$ ) on the impurity peaking factor $R / L_{n_{Z}}$.

To shed some light on the mechanisms at play, the neoclassical background corrections are now applied to the main ions and the trace impurities separately (Fig 3). It is recalled that to derive the analytical expressions for $C_{u}^{N C}$ and the turbulent momentum flux, the neoclassical background has been applied only to the trace impurities or the main ions respectively.

Fig 3 underlines two features of the neoclassical background corrections. When it is applied to the main ions only $\left(F_{n e o, i}\right)$ a small but non zero roto-diffusion is obtained, stemming from the nonzero $k_{\|}$introduced via the neoclassical background. On the other hand, when it is applied to the trace impurities only $\left(F_{n e o, I}\right)$, the modification to the roto-diffusion stems from the $E \times B$ advection in the background rotation gradient as described in Eq. (29) (in this case, the parallel symmetry of the eigenfunction is strictly preserved). The latter mechanism, not present for a Maxwellian background due to the parity in $v_{\|}$, is the dominant one compared to changes in the main ions parallel dynamics $\left(k_{\|} \neq 0\right)$ and provides the most efficient way of generating a nonzero roto-diffusion. The turbulent momentum flux induced by the neoclassical background is only obtained via $F_{n e o, i}$ due to its intrinsic link with symmetry breaking mechanisms. These results support the separation made between main ion and trace impurities dynamics to derive the analytical expressions of section III.
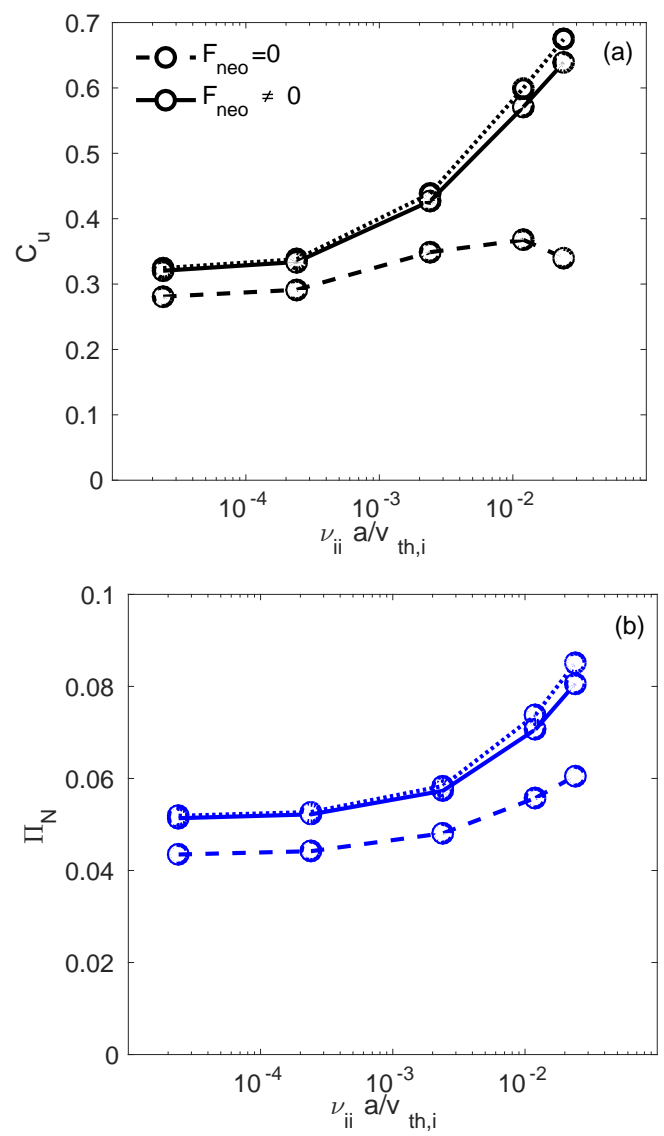

FIG. 4: Roto-diffusion coefficient $C_{u}$ for trace carbon (a) and normalised turbulent ion momentum flux (b) versus the normalised ion-ion collisionality for cyclone base case, with $u=0.2$ and $u^{\prime}=$ 0.7 , with $F_{n e o}=0$ (dashed line) and $F_{n e o} \neq 0$ (full line). The fluxes resulting from the sum of the standard case of Fig 1 and 2 and the $F_{n e o}=0$ case are indicated with a dotted line.

Simulations with typical values of normalised toroidal rotation $u=0.2$ and corresponding gradient $u^{\prime}=0.7$ are shown in Fig 4 to compare the modifications introduced by the neoclassical background to results obtained for a Maxwellian background. To this end, the same scan in collisionality for carbon trace impurities is performed including or not the neoclassical equilibrium correction (applied to all species).

As expected, $C_{u}$ and $\Pi_{N}$ are not zero even with $F_{\text {neo }}=0$, due to the finite values of $u$ and $u^{\prime}$. The impurity and momentum fluxes driven by the neoclassical background are comparable to those driven by the finite rotation, provided the collisionality is large enough. Furthermore, the contributions driven by the neoclassical background and the finite rotation are nearly additive, as demonstrated by summing the fluxes obtained for cases at $u=0$ and $u^{\prime}=0$ (Fig 1$)$ to the $F_{n e o}=0$ cases where $u$ and $u^{\prime}$ are different from zero. This justifies the analyses on cases with $u=u^{\prime}=0$. 


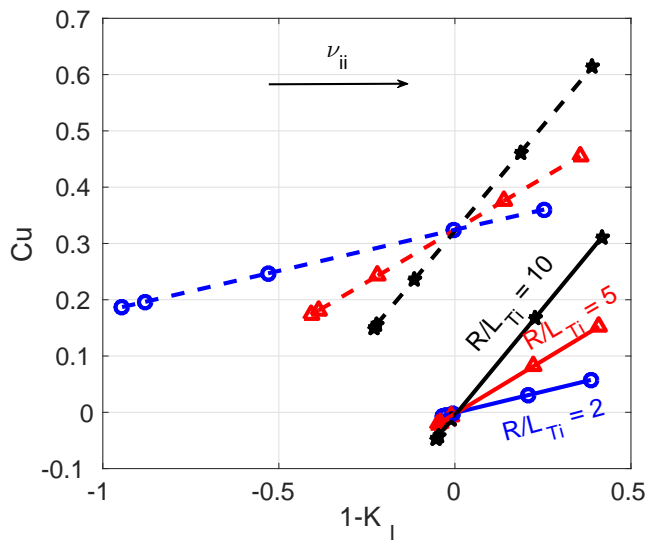

FIG. 5: (color online) Roto-diffusion versus $1-K_{I}$ for $R / L_{T_{i}}=2$ (blue circles), $R / L_{T_{i}}=5$ (red triangles) and $R / L_{T_{i}}=10$ (black pentagrams). Simulations are performed at two values of $R / L_{n}$, $R / L_{n}=0$ (solid lines) and $R / L_{n}=4.4$ (dashed lines). The background gradients are modified in NEO only.

\section{A. Parametric dependencies}

We now scrutinize the qualitative dependencies of $C_{u}^{N C}$ and the neoclassical background induced momentum flux with plasma parameters, underlined in the model of section III. In the following, each of the analytical dependencies are investigated in numerical simulations, varying plasma local parameters independently. Two types of simulations are performed, considering the modifications of the parameters only in NEO (thus modifying the neoclassical background only) or consistently modifying parameters in NEO and GKW. These two approaches are denoted by $\mathrm{NEO}$ and $\mathrm{NEO}+\mathrm{GKW}$ in the figure legends. The fixed parameters are set to the values specified in Table I.

First the qualitative trends obtained analytically with $1-K_{a}$ (collisionality), $R / L_{T_{i}}$ and $R / L_{n}$ are investigated by varying these quantities in NEO only. $K_{i}$ and $K_{I}$ are the flow coefficients (extracted from NEO simulations) for the main ions and the impurities respectively. In Fig 5 , the roto-diffusion coefficient is shown for a scan in collisionality at three values of $R / L_{T_{i}}(2,5,10)$ and two values of $R / L_{n}(0,4.4)$. It is recalled that Eq. (29) predicts a linear dependence of $C_{u}$ on $1-K_{I}$. The slope is directly proportional to $R / L_{T_{i}}$ and shifted by $R / L_{n}$. These qualitative results are recovered in Fig 5 where an increase of the slope is observed with increasing $R / L_{T_{i}}$ (it can be shown that this increase is directly proportional to the increase in $R / L_{T_{i}}$ at constant $\left.R / L_{n}\right)$. Furthermore, cases at $R / L_{n}=0$ show a reversal of the roto-diffusion concomitant with a reversal of $1-K_{I}$. At $R / L_{n}=4.4$, this reversal of $C_{u}$ is no longer observed due to the upshift introduced via the non-zero background density gradient. This upshift also preserves the slopes which are only proportional to $R / L_{T_{i}}$.

A similar investigation for the turbulent momentum flux variations with respect to the collisionality, $R / L_{n}$

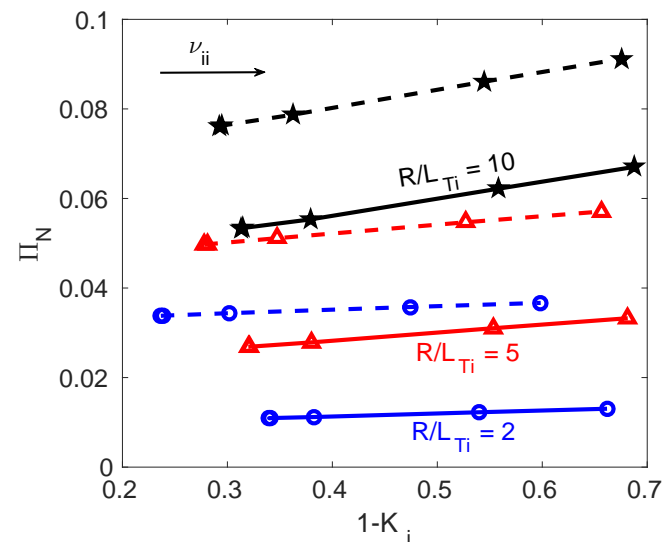

FIG. 6: Normalised turbulent momentum flux versus $1-K_{i}$ for $R / L_{T_{i}}=2$ (blue circles), $R / L_{T_{i}}=5$ (red triangles) and $R / L_{T_{i}}=$ 10 (black pentagrams). Simulations are performed at two values of $R / L_{n}, R / L_{n}=0$ (solid lines) and $R / L_{n}=4.4$ (dashed lines). The $\nabla H$ term has been artificially removed and the background gradients are modified in NEO only.

and $R / L_{T_{i}}$ is performed in Fig 6 . The results are now shown against $1-K_{i}$ and analysed using Eq 27. The term $\nabla H$ has been artificially removed (no second derivatives and no $\left(R / L_{T_{i}}\right)^{2}$ terms) to unravel the analytical linear trends. Again the slopes of the numerically computed momentum flux are directly proportional to $R / L_{T_{i}}$ and shifted upward for increasing $R / L_{n}$. In contrast to the roto-diffusion, the momentum flux is always positive due to the flow coefficient $K_{i}<1$. It can be noted that this flux does not converge toward zero with $1-K_{i}$. This behaviour is due to the nonzero $k_{\|}$generated by the neoclassical equilibrium, which increases with $R / L_{T_{i}}$ and $R / L_{n}$ and not taken into account into Eq 27.

In Fig 7 the impact of $\nabla H$ in the turbulent momentum flux is now assessed (second derivatives are still set to zero) at two collisionalities, $\nu_{i i} a / v_{t h, i}=2.4 \times 10^{-5}$ and at $\nu_{i i} a / v_{t h, i}=2.4 \times 10^{-2}$. It is shown that, in qualitative agreement with $\mathrm{Eq} 27$, the momentum flux is linear with $R / L_{T_{i}}$ provided $\nabla H$ is set to zero and features a quadratic dependency in the opposite case where $\nabla H \neq 0$, even triggering a sign reversal at high $R / L_{T_{i}}$ and low collisionalities. This behaviour is observed in both collisionality regimes.

The background gradients are now scanned consistently in both codes, thus taking into account their direct impact on the turbulence drive. This is performed at two collisionalities (Fig 8 and 9). The parameter space investigated features both Ion temperature gradient (ITG) and trapped electron modes (TEM).

It is observed that roto-diffusion at low collisionality (Fig 8a) is increasing with $R / L_{n}$ but slightly decreasing with $R / L_{T_{i}}$ (negative $1-K_{I}$ ). In contrast, at high collisionality (Fig 8b), it is increasing with both $R / L_{n}$ and $R / L_{T_{i}}$ (positive $1-K_{I}$ ). These results including now the variations of the turbulence drive (gradients modified in GKW as well), are still in qualitative agreement with Eq 


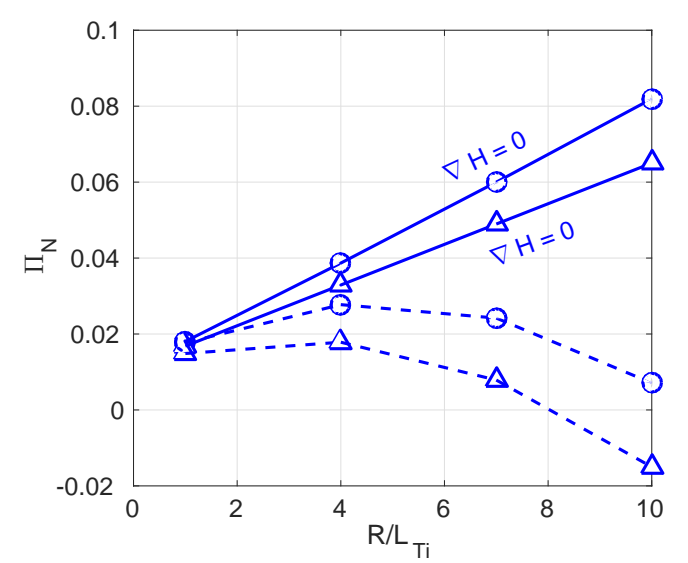

FIG. 7: Normalised turbulent momentum flux versus the logarithmic temperature gradient at low (triangles) and high (circles) collisionalities, $\nu_{i i} a / v_{t h, i}=2.4 \times 10^{-5}$ and $\nu_{i i} a / v_{t h, i}=2.4 \times 10^{-2}$ respectively. Results are shown with (dashed lines) and without (solid lines) $\nabla H . R / L_{T_{i}}$ is modified in NEO only.

29.

As for the turbulent momentum flux, it is increasing with $R / L_{n}$ and $R / L_{T_{i}}$ in both collisionality regimes (Fig $9 \mathrm{a}, 9 \mathrm{~b})$. This behaviour is no longer consistent with Eq.(27) and likely due to a dominant effect of the drive countering the $\left(-R / L_{T_{i}}\right)^{2}$ stemming from the neoclassical background radial gradient.

In Fig 10, a scan in the impurity mass is performed up to $M_{I}=184$ for $Z=10$ and $Z=40$. It is recalled that no toroidal rotation is used in these simulations, thus centrifugal effects are absent in these results.

The model predicts a linear dependency with the mass and none with the charge $Z$. It is seen that this linear trend is recovered in the simulations and a small impact of $Z$ (compared to the mass dependency) is observed especially for high mass impurities. Applying the neoclassical equilibrium correction to the impurities only $\left(F_{n e o, I}\right)$ also leads to a change of $C_{u}$ with $Z$ which is therefore not due to the modification of the parallel dynamics of the main ions.

In Fig 11 and 12 the dependency of the roto-diffusion on the safety factor $q$ and inverse aspect ratio $\epsilon$ are investigated. These dependencies arise from the term $B_{t} / B_{p}$ in the neoclassical flows, which is equivalent to $q / \epsilon$ in circular geometry at low $\epsilon$. The scan in $\epsilon$ is performed in such a way that each parameter is kept constant with respect to Table I.

Roto-diffusion $C_{u}$ is shown to increase linearly with $q$ (Fig 11), which is consistent with the model predictions when this parameter is varied in NEO only. In contrast, the dependency with $1 / \epsilon$ is roughly linear and the slope is different from the one obtained with $q$. This is explained by the fact that changing $\epsilon$ also modifies the flow coefficient $K_{I}$ due to the variation of the trapped particle fraction. Variying $q$ and $\epsilon$ in GKW as well yields the same qualitative behaviour, that is an increase of $C_{u}$
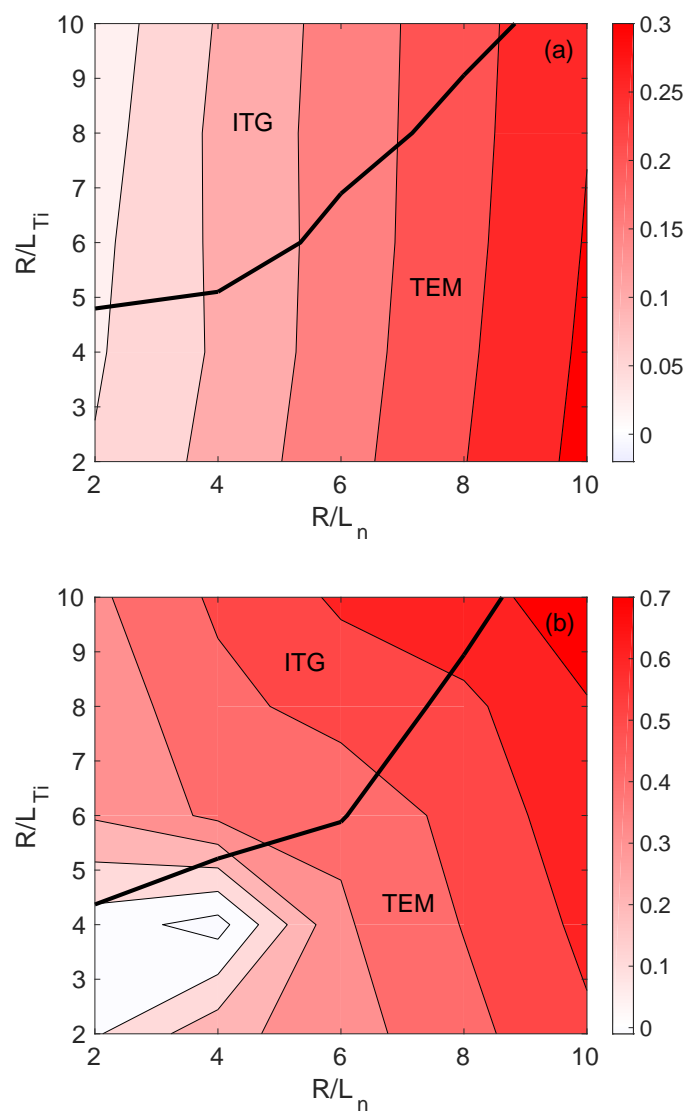

FIG. 8: Coefficient $C_{u}^{N C}$ versus $R / L_{n}$ and $R / L_{T_{i}}$ at $\nu_{i i} a / v_{t h, i}=$ $2.4 \times 10^{-5}$ (a) and $\nu_{i i} a / v_{t h, i}=2.4 \times 10^{-2}$ (b). The black line denotes the limit between TEM and ITG modes.

with the safety factor and the aspect ratio.

On the other hand the behaviour of the turbulent momentum flux is a bit different with respect to these parameters (Fig 12). While linear dependencies are recovered when changing $q$ and $\epsilon$ in NEO only, modifying $q$ in GKW yields a near constant turbulent momentum flux in the parameter range, due to a concomitant reduction of the mean parallel wave vector. Overall, it is shown that $\Pi_{N}$ induced via the neoclassical background is increasing with the aspect ratio $1 / \epsilon$ and roughly constant for typical values of the safety factor $q$.

Finally, the impact of the directions of the toroidal field and plasma current $\left(s_{B}, s_{J}\right)$ on the turbulent momentum and impurity fluxes is investigated. Indeed these directions affect directly the neoclassical flows, and hence the neoclassical background (NEO), but also the drift velocities (GKW). In the description of section II, a simple slab-like geometry has been used, thus not considering all the sign dependencies in the drifts present for more complex geometries. The sign conventions used are the same as those in NEO, that is, positive values corresponding to the counter-clockwise direction when viewed from above.

In Fig 13(a) and (b), a scan in collisionality is performed with different set up of plasma current and 

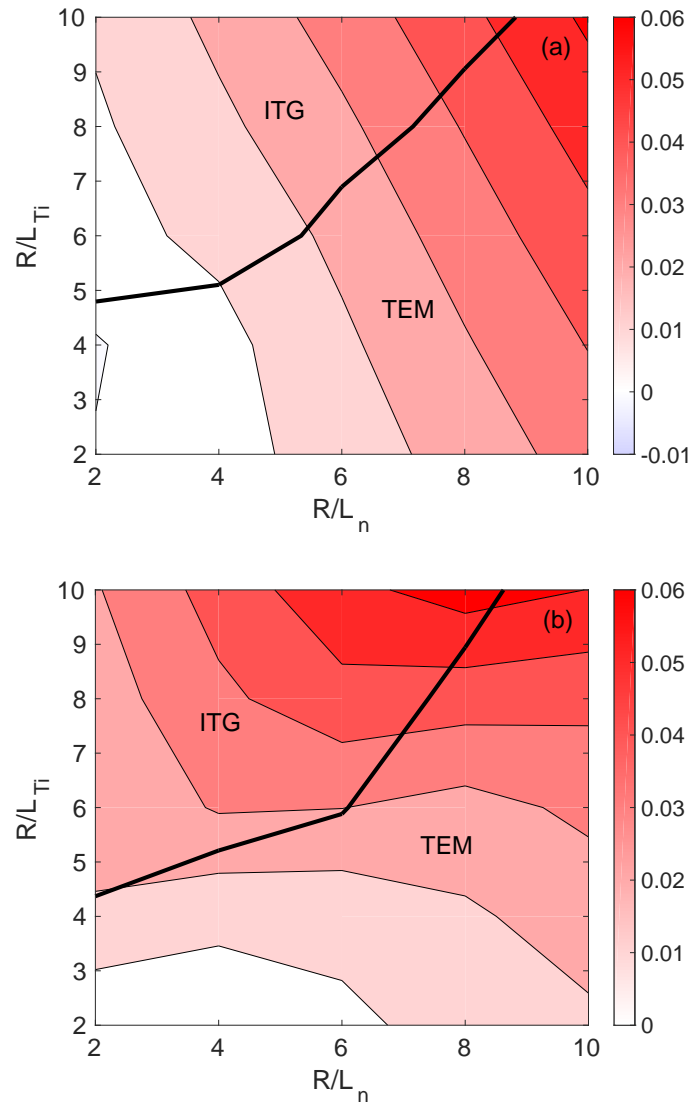

FIG. 9: Normalised ion momentum $\Pi_{N}$ flux versus $R / L_{n}$ and $R / L_{T_{i}}$ at $\nu_{i i} a / v_{t h, i}=2.4 \times 10^{-5}$ (a) and $\nu_{i i} a / v_{t h, i}=2.4 \times 10^{-2}$ (b). The black line denotes the limit between TEM and ITG modes.

toroidal magnetic field signs. It is shown that $C_{u}^{N C}$ is dependent on the direction of the toroidal magnetic field and plasma current, leading to either positive (outward) or negative (inward) contributions. In contrast, the turbulent momentum flux depends on the sign of the plasma current only.

To summarise, the neoclassical background induced roto-diffusion $C_{u}^{N C}$ increases with increasing collisionality, $R / L_{n}$ and $R / L_{T_{i}}$ (only at high collisionalities for the latter, where $1-K_{I}$ is positive), safety factor, aspect ratio, impurity mass and is either inward or outward depending on the respective sign of the plasma current and toroidal magnetic field. As for the turbulent momentum flux, it increases with increasing collisionality, $R / L_{n}$, $R / L_{T_{i}}$, aspect ratio and is dependent on the sign of the plasma current only.

\section{B. Impact of second derivatives and mode frequencies}

Throughout this paper we have considered only one poloidal wave number $\left(k_{\theta} \rho_{i}\right)$ and the profiles used in NEO were produced such that the second derivative of

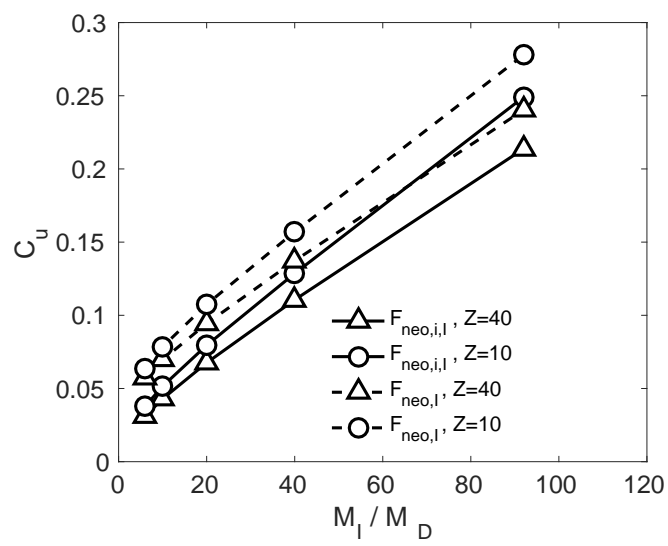

FIG. 10: Roto-diffusion coefficient versus the impurity mass for $Z=10$ and $Z=40$, taking into consideration the neoclassical background correction on the trace impurities only $\left(F_{n e o, I}\right.$, dashed lines) or on all species $\left(F_{n e o, i, I}\right.$, solid lines).

temperature and density profiles were set to 0 . In the following the impact of second derivatives and poloidal wave number on the neoclassical background induced turbulent momentum and impurity transport is investigated for a low collisionality case $\left(\nu_{i i} a / v_{t h, i}=2.4 \times 10^{-5}\right)$. To study these dependencies, some assumptions used to derive 27 and 29 are reconsidered. Indeed second derivatives only enter the perturbed parallel velocity equation. Hence considering uncoupled equations via the condition $k_{\|, N}-4 x_{a} u / Z \sim 0$ yields no effect of such derivatives on the impurity flux in contrast to the momentum flux. To introduce second derivatives and the poloidal wave number dependency into the turbulent transport coefficients (impurity flux), the assumptions $k_{\|, N}-4 x_{a} u / Z \sim 0$ and $\tilde{T}=0$ must be relaxed, to couple the density fluctuation equation either to the temperature fluctuations or to the parallel velocity fluctuations.

In Fig 14 the impact of second derivatives on $C_{u}^{N C}$ and $\Pi_{N}$ is shown for the Cyclone base case, varying the parameters $\alpha_{n}$ and $\alpha_{T_{i}}$ defined below:

$$
\begin{aligned}
\alpha_{n} & =\frac{R^{2}}{n} \frac{\partial^{2} n}{\partial r^{2}} \\
\alpha_{T_{i}} & =\frac{R^{2}}{T_{i}} \frac{\partial^{2} T_{i}}{\partial r^{2}}
\end{aligned}
$$

The neoclassical correction has been applied either to the trace impurities or to the main ions. It appears that, as found in the fluid model, second derivatives have no impact on $C_{u}$ in cases where $k_{\|, N}-4 x_{a} u / Z=0$, that is when the corrections are applied only to the trace species. Introducing the correction on the main ions generates a nonzero $k_{\|}$which then leads to a linear dependency with the second derivatives. It is also observed that the slopes of the linear dependencies of $C_{u}$ with $\alpha_{n}$ and $\alpha_{T_{i}}$ are different, due to the $1-K_{a}$ in front of the $\alpha_{T_{i}}$ term (see Eq 17 ). The same observations can be made for the 

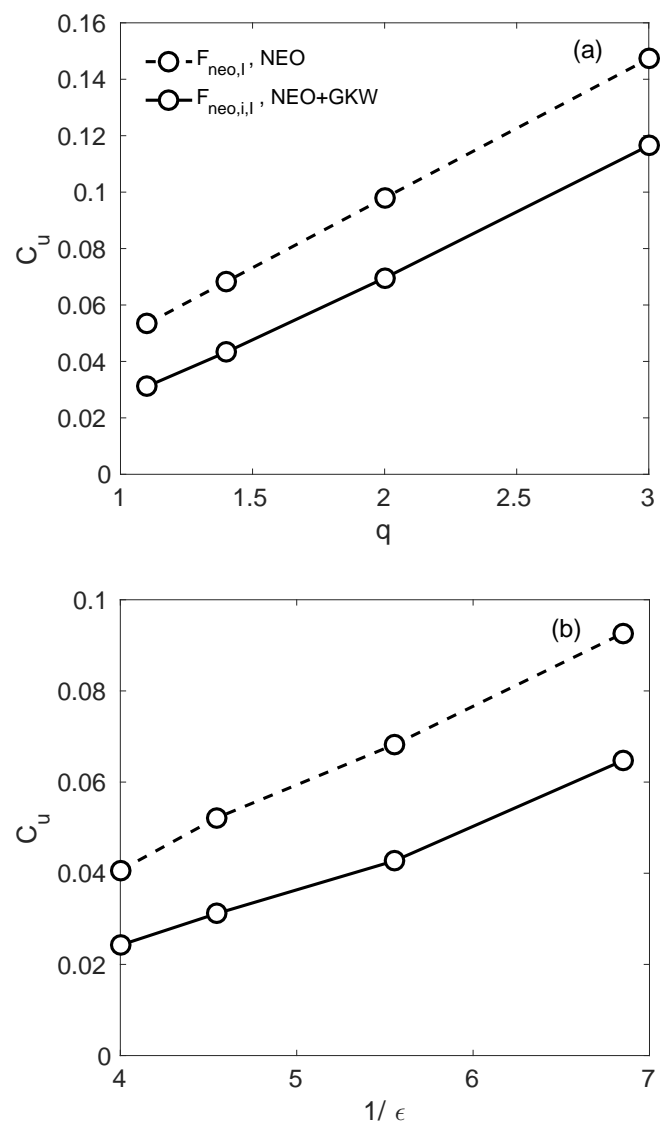

FIG. 11: Roto-diffusion versus the safety factor $q$ (a) and the inverse aspect ratio $\epsilon(\mathrm{b})$.

turbulent momentum flux (Fig 14(b)). Furthermore, the dependencies are not symmetric with respect to $\alpha_{n, T}$. The generated roto-diffusion and momentum flux crosses zero for positive values of the second derivatives and at much higher values for $\alpha_{T}$ than for $\alpha_{n}$. This is expected from Eq. (27) and due to the compensation of the terms $\left(R^{2} / T_{i}, n\right) \partial^{2} T_{i}, n / \partial r^{2}$ and $-\left(R / L_{T_{i}, n}\right)^{2}$.

Looking now at the poloidal wave number dependency, the condition $\tilde{T}=0$ is relaxed and yields the following roto-diffusion coefficient (using $\mathrm{Eq} 16$ and 18):

$$
C_{u}=f_{C_{u}} \rho_{*} \frac{B_{t}}{B_{p}} \frac{m_{I}}{m_{i}}\left[\frac{R}{L_{T_{i}}}\left(1-K_{I}\right)+\frac{R}{L_{n}}\right]
$$

with:

$$
f_{C_{u}}=1-\frac{\frac{12 \omega_{R}}{\omega_{D} Z\left|3 \omega_{N}+\frac{14}{Z}\right|^{2}}+\frac{40}{Z^{2}\left|3 \omega_{N}+\frac{14}{Z}\right|^{2}}}{1+\frac{24}{Z^{2}\left|3 \omega_{N}+\frac{14}{Z}\right|^{2}}}
$$

In Fig. 15, $C_{u}$ and the coefficient $f_{C u}$ are computed and shown versus the poloidal wave number at $\nu_{i i} a / v_{t h, i}=$ $2.4 \times 10^{-5}$. The trace impurities used in these simulations have $m_{I} / m_{i}=10$ and $Z=1$ or 10 . This change in
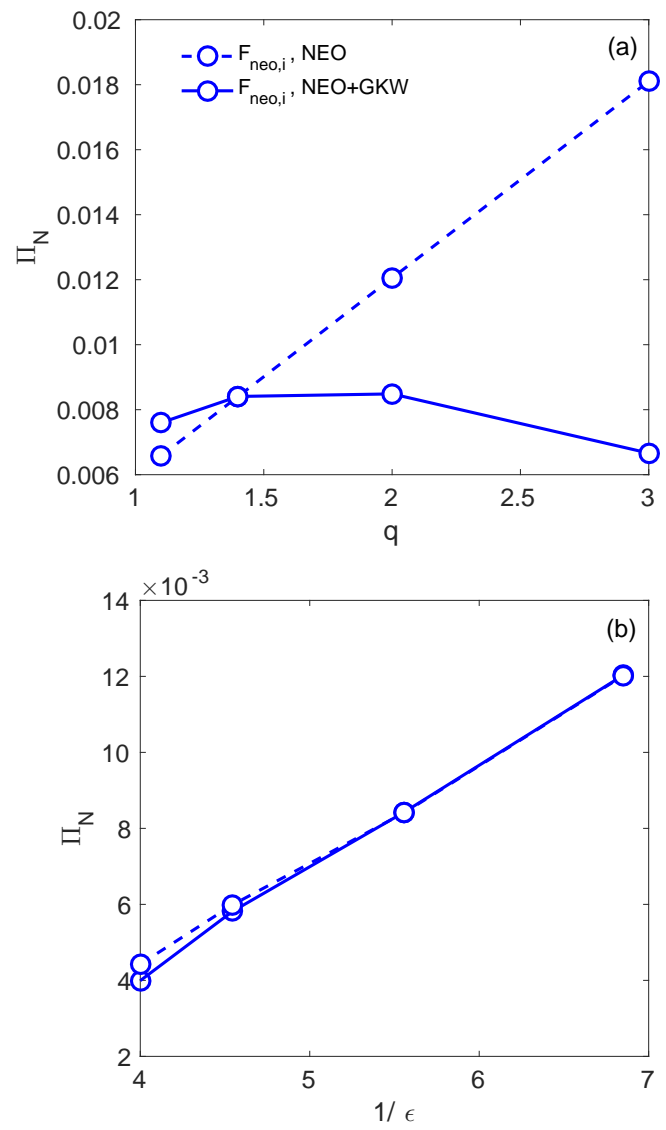

FIG. 12: Normalised turbulent momentum flux versus the safety factor $q(\mathrm{a})$ and the inverse aspect ratio $\epsilon$ (b).

$Z$ allows us to disentangle regimes where $f_{C u} \sim 1$ and $f_{C u} \neq 1$.

Going from Ion Temperature gradient (ITG) to Trapped Electrons mode (TEM) instabilities, i.e. changing the sign of the mode frequency $\hat{\omega}_{R}$, the coefficient $f_{C u}$ decreases together with the neoclassical background correction induced roto-diffusion. This behaviour is particularly strong for low $Z$ impurities (extreme case here $Z=1)$. The amplitude of this reduction is limited at larger $Z$ yielding a coefficient $f_{C u} \sim 1$ regardless of the main instability type. In the ITG spectral range, the roto-diffusion follows the trend of $f_{C_{u}}$, i.e. it increases with the poloidal wave number for $Z=1$ and decreases for $Z=10$. It is important to note that the fluid model for which $f_{C u}$ is derived is not tailored for TEM studies as it does not encompass trapped particles dynamics. However it allows one to assess that, qualitatively, the roto-diffusion introduced by the neoclassical background corrections is expected to be of similar amplitude in the ITG/TEM domain for modest to high $Z$ impurities whereas it is expected to be lower for low $Z$ impurities in the TEM regime which is confirmed numerically. 

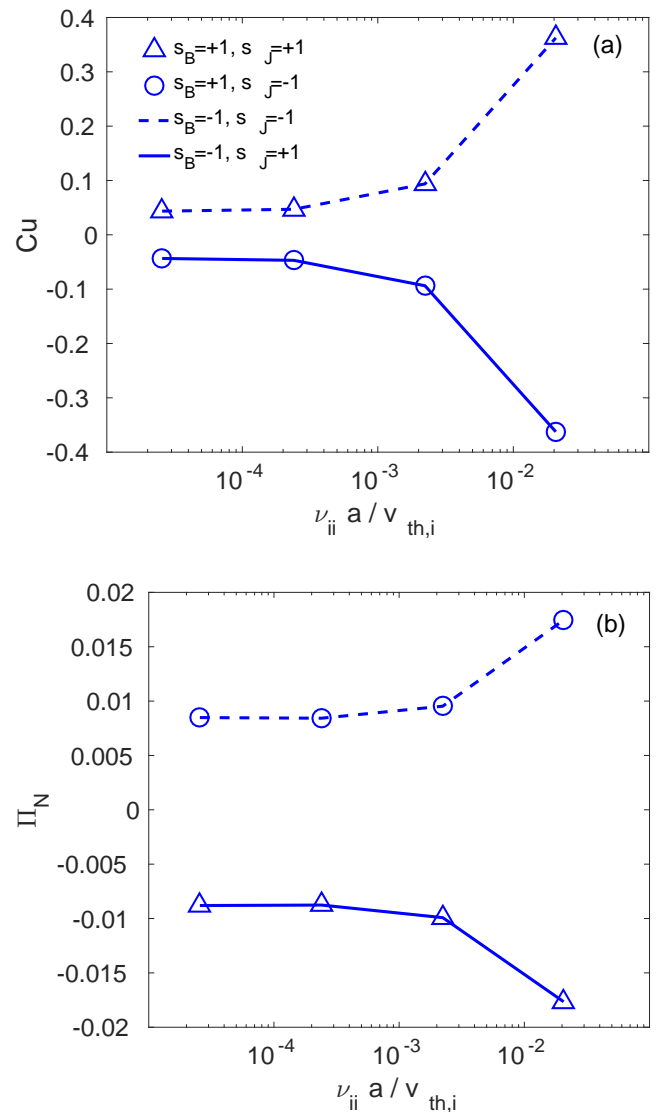

FIG. 13: Roto-diffusion coefficient and normalised turbulent momentum flux versus the normalised ion-ion collisionality for different combinations of the signs of the toroidal magnetic field and plasma current.

\section{CONCLUSIONS}

The impact of the neoclassical background on turbulent fluxes has been investigated via both gyrokinetic simulations and a fluid model making use of a Laguerre polynomials expansion of the first order neoclassical distribution function. While important results already found in the literature for neoclassical background induced turbulent momentum flux are recovered (residual turbulent momentum flux at low collisionality, collisionality dependence), a new impurity transport mechanism is highlighted. Numerical simulations and analytical expressions were used to identify the origin of the dependencies of this mechanism with plasma parameters and to benchmark the implementation of the neoclassical background in the gyrokinetic code GKW [16].

It is found that two main mechanisms are contributing to the generation of roto-diffusion via the neoclassical background. One is linked to the change of the parallel symmetry of the turbulent eigenfunction through equilibrium corrections on the main ions. This mechanism is also responsible for modifications of the turbulent momentum fluxes $[13,14]$. The second one,
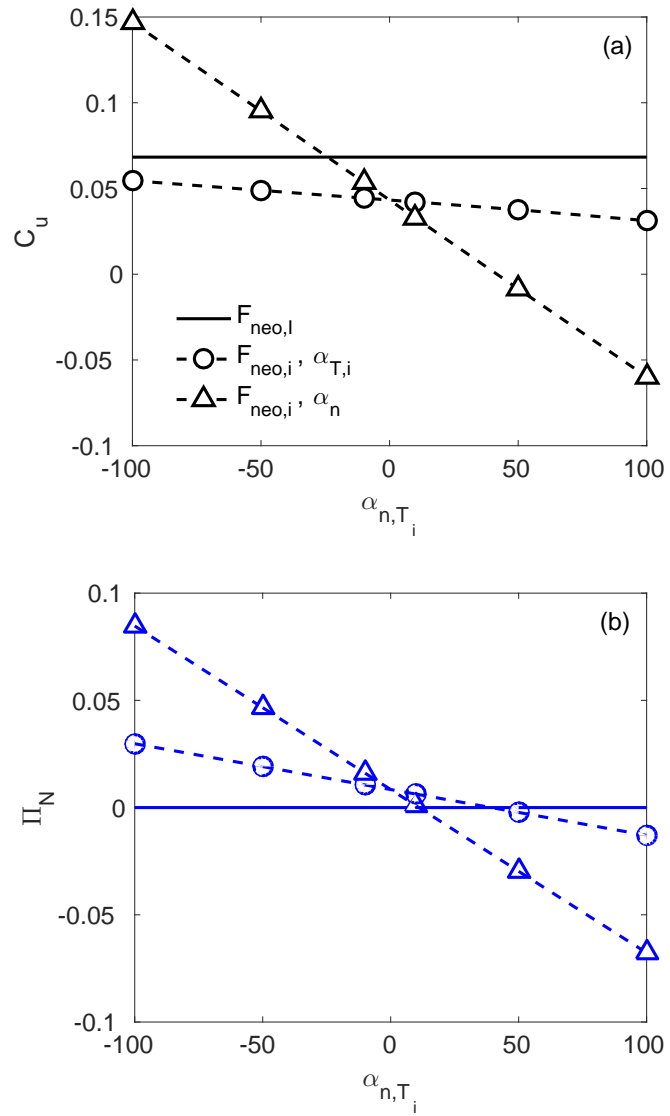

FIG. 14: Roto-diffusion $C_{u}$ (a) and normalised ion momentum flux (b) versus parameters $\alpha_{n, T_{i}}=\left(R^{2} / n, T_{i}\right) \partial^{2} n, T_{i} / \partial r^{2}$ at $\nu_{i i} a / v_{t h, i}=2.4 \times 10^{-5}$. Results are shown for neoclassical background corrections applied to the trace impurities alone (plain lines) and to all species (dashed lines).

which is found dominant for turbulent impurity transport compared to the former, is non-zero contributions from the velocity space integrals of $v_{\|} F_{n e o}$ of the impurities, stemming from the asymetry in the parallel velocity space of the first order neoclassical distribution function. For roto-diffusion, this implies the parallel streaming, Coriolis drift and $E \times B$ advection in the toroidal rotation background gradient entering the perturbed fluid density equation. These integrals generate a nonzero roto-diffusion even in the absence of a coupling between the impurities density and parallel velocity fluctuations which is a new feature compared to the standard Maxwellian background. For momentum transport it implies the integrals related to the $\nabla B$ and curvature drift, the $E \times B$ advection in the temperature and density background gradients and their second derivatives, entering the perturbed fluid parallel velocity.

In an ideal set of parameters (no toroidal rotation and corresponding gradient) and for turbulent trace impurity transport only, these two distinct mechanisms can be decoupled, applying the neoclassical background either on the main ions or the trace impurities. In this 

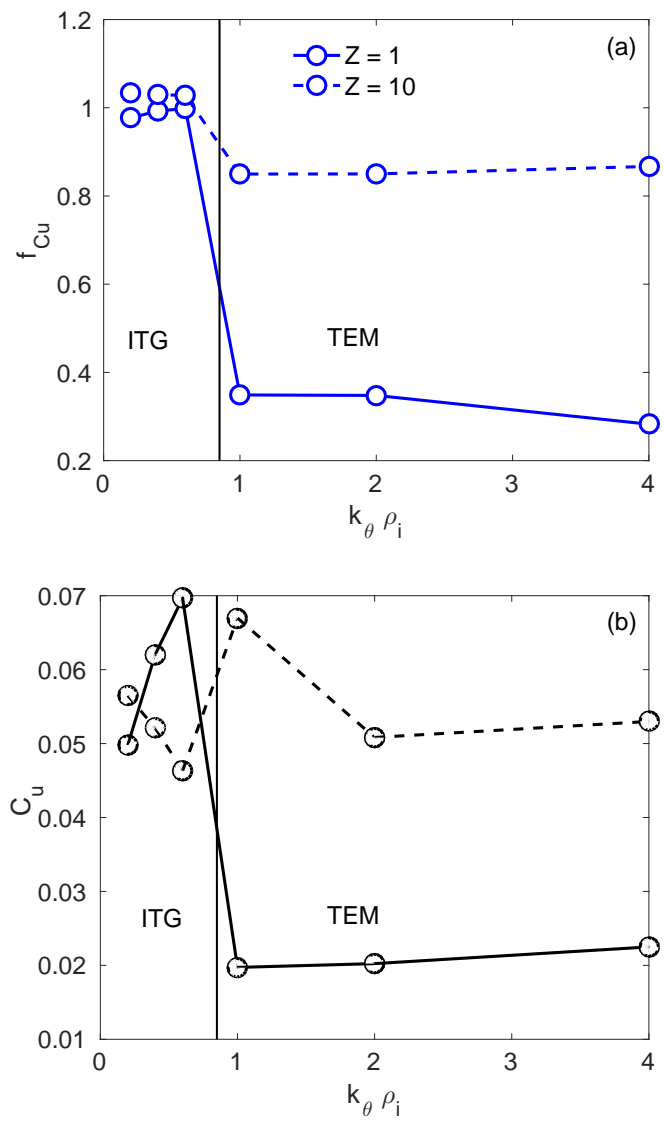

FIG. 15: Coefficient $f_{C_{u}}$ (Eq. (36)) (a) and the roto-diffusion coefficient $C_{u}$ (b) versus the normalised poloidal wave number. ITG and TEM instabilities for the most unstable mode are also delimited.

framework, the fluid model is found to qualitatively reproduce the numerical simulations. Furthermore, it is shown that the second mechanism yields a roto-diffusion coefficient that increases with increasing collisionalities, $R / L_{T_{i}}, R / L_{n}$, impurity mass, $q / \epsilon$ and that is related to the second derivatives of the density and temperature profiles. It is therefore expected to have strong impact for modest to high mass impurities (provided turbulent transport is non-negligible compared to neoclassical transport), in presence of internal transport barriers or towards the edge of tokamak plasmas.

Finally, the impact on roto-diffusion is dependent on the direction of the plasma current and toroidal magnetic field, yielding either inward or outward contributions. This fingerprint of the impact of the neoclassical background on impurity turbulent transport could be used for an experimental identification.

\section{Acknowledgments}

W. A. Hornsby and C. Angioni aknowledge very fruitful discussions with F. I. Parra and M. Barnes.

\section{Appendix A: Impact of the numbers of Laguerre polynomials}

The quantitative impact of the number of Laguerre polynomials in assessing the roto-diffusion induced by the neoclassical background is investigated through a scan in collisionality for the standard case described Table I. The characteristic collisionality dependence in $1-K_{I}$ is then shown for different numbers of Laguerre polynomials in Fig 16.

To perform these simulations, the same number of polynomials are used in NEO (5), and a limited number are then read in GKW to reconstruct the distribution function. Going from one to two Laguerre polynomials leads to a quantitative change in the turbulent roto-diffusion at all collisionalities. Further increasing the numbers of Laguerre polynomials up to 5 does not change noticeably the results (for these parameters). The linear trend with $1-K_{I}$ is recovered for any number of polynomials.

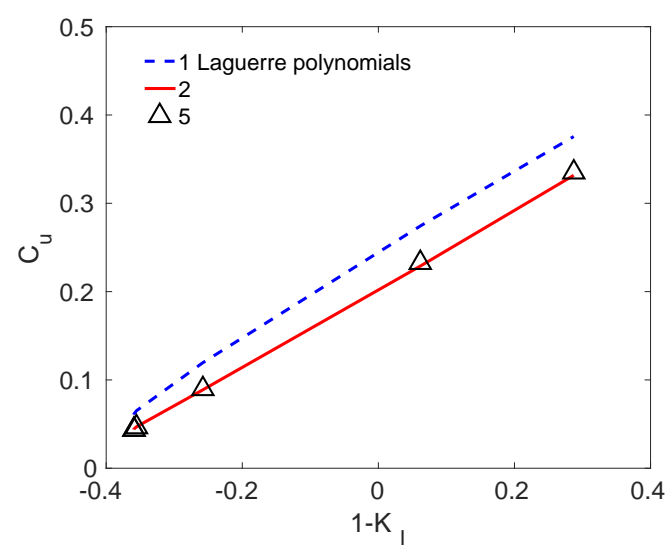

FIG. 16: Roto-diffusion calculated for a carbon trace species versus $1-K_{I}$ with different numbers of Laguerre polynomials used in GKW. The analytical expression 29 is also shown.

The numerically accurate neoclassical distribution functions can also be directly compared to reconstructed distribution functions in velocity space using either one or two Laguerre polynomials, that is, using Eq. (5) with the parallel flows computed from NEO.

In Fig 17 two perturbed distribution functions are extracted from NEO (reconstructed from 5 Laguerre polynomials and 17 Legendre polynomials) at low collisionality $\left(\nu_{1}=\nu_{i i} a / v_{t h, i}=2.4 \times 10^{-5}\right)$ and higher collisionality $\left(\nu_{2}=\nu_{i i} a / v_{t h, i}=2.4 \times 10^{-2}\right)$. It is seen that the low collisionality case Fig 17(a) presents shapes at higher energy structures in the velocity space $\left(v_{\|}, v_{\perp}\right)$ than the high collisionality case Fig 17(b).

A distribution function is reconstructed using two (c) and one (d) Laguerre polynomials respectively. It is shown that the qualitative picture in the velocity space of the numerically accurate distribution functions can be recovered using at least 2 Laguerre polynomials at low 
collisionality and one at high collisionality.

To summarise, the qualitative shape in velocity space of the neoclassical distribution function $F_{n e o}$ is well recovered at high collisionalities with one Laguerre polynomials whereas 2 Laguerre polynomials are necessary at low collisionalities. Furthermore going from one to two Laguerre polynomials changes quantitatively the induced roto-diffusion in the whole range of collisionalities but keeps the linear trend with $1-K_{I}$ thus justifying to some extent the qualitative comparisons between the fluid model (retaining only one polynomial) and the numerical simulations performed in this paper.
[1] M. R. Wade, W. A. Houlberg, and L. R. Baylor, Physical Review Letters 84, 282 (2000)

[2] F. J. Casson et al, Nuclear Fusion 53, 063026 (2013)

[3] C. Angioni et al, Nuclar Fusion 54, 083028 (2014)

[4] F. J. Casson et al, Plasma Physics and Controlled Fusion 57, $014031(2015)$

[5] A. Skyman, L. Fazendeiro, D. Tegnered, H. Nordman, J. Anderson and P. Strand, Nuclear Fusion 54, 013009 (2013)

[6] H. Nordman, A. Skyman, P. Strand, C. Giroud, F. Jenko, F. Merz, V. Naulin, T. Tala, and the JET-EFDA Contributors, Plasma Physics and Controlled Fusion 53, 105005 (2011)

[7] D. R. Mikkelsen et al, Physics of Plasmas 21, 082302 (2014)

[8] S. Sudo, Plasma Physics and Controlled Fusion 58, 043001 (2016)

[9] G. Dif-Pradalier, V. Grandgirard, Y. Sarazin et al, Physical Review Letters 103, 065002 (2009)

[10] M. Oberparleiter, F. Jenko, D. Told, H. Doerk and T. Görler, Physics of Plasmas 23, 042509 (2016)

[11] Y. Idomura, Physics of Plasmas 21, 022517 (2014)

[12] F. I. Parra and M. Barnes, Plasma Physics and Controlled Fusion 57, 045002 (2015)

[13] M. Barnes, F. I. Parra, J. P. Lee, E. A. Belli, M. F. F. Nave, and A. E. White, Physical Review Letters 111, 055005 (2013)

[14] J. P. Lee, M. Barnes, F. I. Parra, E. Belli, and J. Candy, Plasma Physics and Controlled Fusion 57, 125006 (2015)

[15] J. P. Lee, F. I. Parra and M. Barnes, Nuclear Fusion 54, $022002(2014)$

[16] W. A. Hornsby et al, On the effect of neoclassical flows on intrinsic momentum in ASDEX Upgrade Ohmic L-mode plasmas, submitted to Nuclear Fusion (2016)

[17] Y. Camenen, A. G. Peeters, C. Angioni, F. J. Casson, W. A Hornsby, A. P. Snodin, and D. Strintzi, Physics of Plasmas 16, 012503 (2009)

[18] C. Angioni, Y. Camenen, F. J. Casson, E. Fable, R. M. McDermott, A. G. Peeters, and J. E. Rice, Nuclar Fusion
52, $114003(2012)$

[19] E. A. Belli and J. Candy, Plasma Physics and Controlled Fusion 51, 075018 (2009)

[20] E. A. Belli and J. Candy, Plasma Physics and Controlled Fusion 54, 015015 (2012)

[21] F. L. Hinton and S. K. Wong, Physics of Fluids 28, 3082 (1985);

[22] A. G. Peeters, Y. Camenen, F. J. Casson, W. A. Hornsby, A. P. Snodin, D. Strintzi, G. Szepezi, Computer Physics Communications 180, 2650 (2009)

[23] P. Helander and D. J. Sigmar, Collisional Transport in Magnetized Plasmas (Cambridge University Press, 2001)

[24] Y. B. Kim, P. H. Diamond, and R. J. Groebner, Physics of Fluids B 3, 2050 (1991)

[25] S. Nishimura, H. Sugama, H. Maaßberg, C. D. Beidler, S. Murakami, Y. Nakamura, and S. Hirooka, Physics of Plasmas 17, 082510 (2010)

[26] S. P. Hirshman and D. J. Sigmar, Nuclear Fusion 21, 1079 (1981)

[27] E. A. Belli and J. Candy, Plasma Physics and Controlled Fusion 50, 095010 (2008)

[28] A. G. Peeters, D. Strintzi, Y. Camenen, C. Angioni, F. J. Casson, W. A. Hornsby, A. P. Snodin, Physics of Plasmas 16, 042310 (2009)

[29] Y. Camenen, F. J. Casson, P. Manas, and A. G. Peeters, Physics of Plasmas 23, 022507 (2016)

[30] A. M. Dimits, Physics of Plasmas 7, 969 (2000)

[31] R. L. Miller, M. S. Chu, J. M. Greene, Y. R. Lin-Liu and R. E. Waltz, Physics of Plasmas 5, 973 (1998)

[32] C. Angioni, R. Dux, E. Fable, A. G. Peeters and the ASDEX Upgrade Team, Plasma Physics and Controlled Fusion 49, 2027 (2007)

[33] C. Angioni, E. Fable, M. Greenwald, M. Maslov, A. G. Peeters, H. Takenaga, and H. Weisen, Plasma Physics and Controlled Fusion 51, 124017 (2009)

[34] C. Angioni, and A. G. Peeters, Physical Review Letters 96, 095003 (2006) 

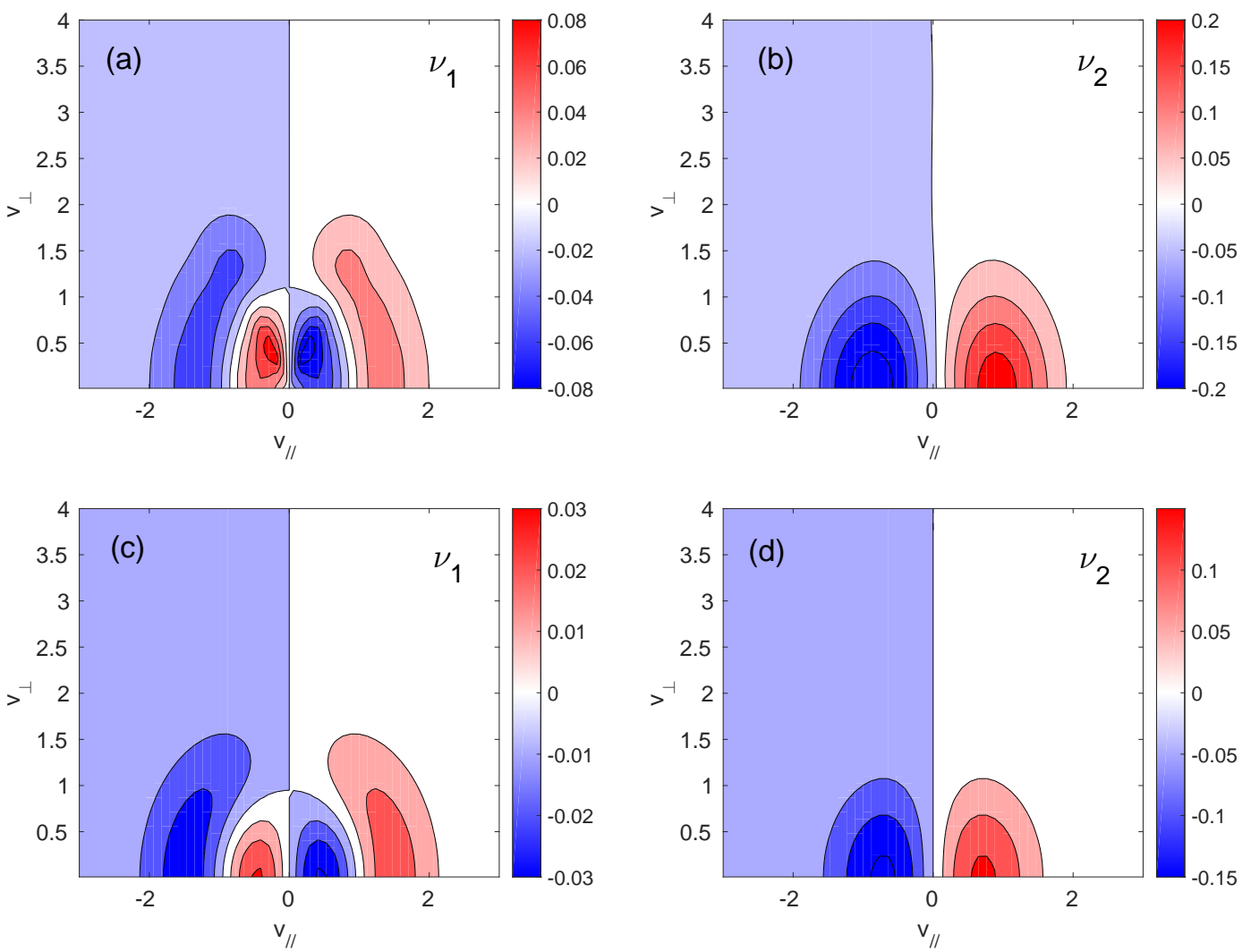

FIG. 17: First order distribution functions extracted from NEO at $\nu_{1}=\nu_{i i} a / v_{t h, i}=2.4 \times 10^{-5}(\mathrm{a}, \mathrm{c})$ and $=\nu_{2}=\nu_{i i} a / v_{t h, i}=2.4 \times 10^{-2}$ $(\mathrm{b}, \mathrm{d})$ compared to reconstructed distribution functions using two and one Laguerre polynomials (c, d respectively) in velocity space $v_{\|}, v_{\perp}$. 